\title{
Application and Development Trend of Marine Biotechnology
}

\author{
Aihwa Wang \\ School of Life Sciences, Qingdao Agricultural University, Qingdao Shandong, 266109, China
}

\begin{abstract}
Key words: Marine biotechnology, Application, Development trend.
\end{abstract}
\begin{abstract}
In China, marine biotechnology industry has become a key field of national economic development during the $12^{\text {th }}$ Five-Year Plan. China has to enhance the development of marine biotechnology due to its large population and constant increase of grain price. As mariculture develops rapidly in the economic development of China, many problems exist inevitably in the development process, such as worsening marine environment, emergence of coastal structure and chaotic management, which seriously influence the development of marine life. Therefore, it is required to enhance the development of high technologies and then improve the benefit of marine industry.
\end{abstract}

\section{Introduction}

Currently, marine biotechnology shows a booming trend in development and utilization in China. With the development of high and new technologies such as genomics and proteomics, Chinese biotechnology has gradually developed towards ocean field. Marine biotechnology, also called as blue biotechnology, involves marine biological genetic engineering, cell engineering and biochemical engineering etc. and meanwhile includes the development of marine life etc. ${ }^{[1]}$ In the current stage, ocean plays a vital role in the sustainable development of coastal countries. Various countries have gradually attached great importance to marine biotechnology which has realized high-speed development in India, Mexico and Southeast Asia region. China also attaches great importance to the development of this technology. It is discussed in detailed below.

\section{General situation of marine biotechnology}

Currently, marine biotechnology has realized brand-new development in various countries, mainly manifested as below: First, various countries have made explorations and conducted research and development constantly, developed new species and applied them to aquiculture. Second, relevant staffs have created higher value for human beings through deep processing of marine resources under the support of marine biotechnology. Third, marine biotechnology system has been established, which enables marine biotechnology to be utilized reasonably in the development of marine biological resources.

\section{Analysis on Development Status of Marine Biotechnology}

Human beings are always curious about ocean which is filled with unknown things not only due to its mystery but also because of its role as great treasury of biological resources. Ocean has continued the history of civilization of human beings and provided countless living resources for human beings. Currently, human beings have constantly consumed land resources. Limitless yearning for and uninterrupted exploitation of resources have made them shift to ocean gradually. The development of marine biotechnology has gradually become a strong and inevitable wave in the world as well as one of the important means taken by various countries in order to resolve the problem of resource shortage, population expansion and environmental degradation.

With marine biological resources as research object, marine biotechnology mainly conducts purposeful transformation, improvement and artificial cultivation specific to marine life, produces, 
industrializes and economizes specific products and forms an economic industrial chain based on ocean. As an important branch of biotechnology industry, marine biotechnology directly reflects the actual development status of biotechnology industry to a certain extent. ${ }^{[2]}$ However, this technology is greatly challenging in the process of research, development and application due to its complicated environment in the research process, including the richness and diversity of marine biological resources and weak study on genetic basis, but its contributions to the future development of human society are extremely considerable, which makes people willing to input energy, financial resources and human power to study it for the purpose of creating a realistic economic application mode mainly involving marine biotechnology. It is not difficult to see from current achievements of the development of marine biotechnology that it is involved in many fields such as biological pharmacy, medical treatment, nanotechnology, functional food, biochemistry and ecological restoration, including artificial mariculture work with marine biological inherited character as basic scientific basis. This makes China form a certain scale in the development of marine agriculture which can even be comparable with powerful nations such as USA and Japan. Moreover, China has reached an advanced level in terms of technical research and development cost input. According to statistics, the total output value of global marine biotechnology industry reached 78 billion US dollars in 2015, including an output value of 20 billion US dollars contributed by China. It proves that marine biotechnology industry has become an important economic industry worth attention in China and even the world in the next decades.

China issued the Decision on Accelerating Cultivation and Development of Strategic Emerging Industry in 2010, which fully specified scientific research and development of marine biotechnology products and industrialized development direction. After the $12^{\text {th }}$ Five-Year Plan, State Oceanic Administration has issued new specifications such as Bioindustry Development Planning ${ }^{[3]}$ and National Marine Affairs Development Planning jointly with the State Council and specifically pointed out the importance of marine biotechnology for future development of biotechnology industry. The development and utilization of marine biological genetic resources and bioactive substances have formed a certain scale. Functional food and pharmaceutical industry mainly involving marine life are advancing steadily and rapidly. Many marine drugs and health care products have been approved for sale. For example, "artificial skin" technology mainly involving skin tissue repair has reached the international advanced level. Moreover, formal clinical trials of a series of new anti-cancer, anti-tumor and anti-AIDS drugs mainly involving marine biotechnology have proved the key of the development and application of marine biotechnology. It has strongly driven the advance of national economy.

\section{Research field of marine biotechnology}

Marine biotechnology is another biotechnology following red, green and white biotechnologies. Red biotechnology is applied to medical field. Green biotechnology is applied to agricultural field and white biotechnology is applied to industrial production. According to the investigation and research of relevant scholars, biotechnologies represented by different colors have different research scopes. The research scope of red biotechnology is health, medical treatment and diagnostics. That of green biotechnology is environment, agriculture, biofertilizer and geomicrobiology. That of white biotechnology is gene-based bioindustries ${ }^{[4]}$. That of marine biotechnology, i.e. blue biotechnology, is aquaculture and coastal and marine biotechnology. The above shows that the research on marine biotechnology involves many aspects, mainly including the development of good breeding varieties, the utilization of special functions of marine life and marine life utilization system. In the gradual development of molecular biological technology, natural products of marine life and aquaculture have gradually become the research hotspot of marine biotechnology.

\section{Application of marine biotechnology}




\section{Marine aquaculture}

According to statistics, marine aquaculture is the primary industry in marine production in China, which occupies half of the marine production industry in China. In fact, marine aquaculture mainly promotes the growth of aquatic products through biotechnology and cultivates high-yield varieties with strong anti-adversity ability while guaranteeing their health status. Early in 1985, China published research achievements on transgenic fish, mainly including trout, Atlantic trout, salmon, tilapia, zebrafish, Crucian carp and catfish. The gene transferred to trout is growth hormone gene which is mainly applied to aquaculture. That transferred to Atlantic trout is growth hormone gene which is mainly applied to aquatic products. That transferred to salmon is also GH gene which is mainly applied to scientific aquaculture research. That transferred to zebrafish is yellow fluorescent protein and RFP and YFP genes which are mainly applied to the study of observation and science. That transferred to tilapia is GFP and GH genes which are mainly applied to the study of aquaculture and science. The gene transferred to catfish is simple, i.e. growth hormone gene which is mainly applied to scientific research. That transferred to Crucian carp is exogenous GH gene which is mainly applied to scientific research and aquaculture. In 2010, relevant researchers disclosed the news that US Food and Drug Administration was considering approving Atlantic trout to appear on the market ${ }^{[5]}$.In addition, the research on marine algae engineering mainly focuses on the establishment and regulation of gene transfer system. For example, kelp, waist band and nori can be expressed through GUS. Moreover, molecular biotechnology can also be applied to the research on algae system.

Marine biotechnology has also been applied to aquatic product processing field. Its application has gradually expanded to articles of daily use and industry from original food. Meanwhile, it can also conduct deep processing and division of fish, accelerate the growth speed of transgenic fish and then improve their yield. It can improve the use ratio of fishing acquisition through the intake of biologic chain.

\section{Development of natural products of marine life and active substances}

A lot of research data show that marine life has good anti-tumor activity and thus is the hotspot of development in the world. Marine life contains the following natural active substances: polyether Gymonocin B, palytoxin and Phakellins alkaloid etc. Polyether Gymonocin B mainly comes from seaweeds and belongs to macrolides alkaloid. Palytoxin mainly comes from actiniae and belongs to toxins compound. Phakellins alkaloid comes from sponge Acanthella costata and belongs to alkaloid.

Macrolides alkaloid has certain anti-tumor activity and has clear effect for soft tissue cancers at late stage such as the therapy of rectal carcinoma, lung cancer and breast cancer. There are clear introductions in relevant research report. In the clinical research of USA on stage III breast cancer, many marine polyether compounds have been found in marine metabolites such as Tian. Besides natural products of anti-tumor activity, they also have anti-inflammatory action. In marine natural products of cerebrocardiac vascular pharmacological activity, PTX has an action strength 100 times of that of common angiotensin. In addition, sponge Acanthella costata has been extracted from other natural products of biological activity, which is an adrenal agonist that can be effectively applied to medical development.

\section{Protection of marine ecological environment}

Currently, biotechnology plays a vital role in the monitoring of marine ecological environment. Marine ecological system can be promoted favorably through biotechnology. Especially in the exploration and development of marine energy resources and marine environmental modification, the application of biotechnology can effectively eliminate marine oil pollution and reduce environmental pollutants.

The application of biosensor technology in the monitoring of marine environment can effectively detect methacrifos in seawater and clearly get the pollution of seawater by pesticide. In the ocean, 
some substances resistant to pollution have strong enrichment of pollutants. It is required to make full use of biomarker and use it as a tool for early warning of environmental pollution when evaluating biological toxicity in time.

\section{Biological nitrogen fixation}

Biological nitrogen fixation belongs to basic recycling substances in the biosphere. Half of them are realized through marine nitrogen-fixing microorganism. Researches of a lot of scholars prove that biological nitrogen fixation plays a vital role in the sustainable development of ocean and has profound influence on the development of productivity of ecological system. Biological nitrogen fixation can act in both oligotrophic and eutrophic sea areas. Marine nitrogen-fixing microorganism has extraordinary significance in the whole marine ecological system, which might gradually become the cutting-edge field of marine biotechnology.

\section{Prospect on development trend}

Ocean contains rich bioactive substances. Ocean can be considered as the resource library with the greatest potential on the earth. As ocean can provide high-quality protein substances and resolve the dilemma faced by human beings to a great extent, marine biotechnology has the widest development space and prospect in future biotechnology development.

The research on marine biotechnology has started late in China, but still achieved great results. Marine biotechnology provides guarantee for the innovation of basic life science, especially molecular biotechnology. It has been clearly specified in IMBC research conference that the development of marine biotechnology will be enhanced in the next few years, especially in biological products of marine natural products, marine drugs and marine environmental protection.

\section{Conclusion}

In conclusion, we should fully apply biotechnology to the development and protection of ocean and define the realization of modernized production of marine organism as the fundamental purpose and development objective in future development so as to provide the most effective marine products for people and promote the economic development of China.

\section{References}

[1] Chen Liguo. Application and Prospect of Marine Biotechnology. Beijing Agriculture, 2016(3):171-171,172.

[2] Tong Xuehong. Explorations on Experimental Teaching Mode of Marine Biotechnology Major. Teaching of Forestry Region, 2013(7):8-9.

[3] Jiao Yanling, Liu Changqiu. Thinking on Strengthening of Legislation for Marine Biotechnology Industry in China. Journal of Henan Business College, 2015(2):64-70.

[4] Marine Biotechnology Achievements of Lei Li Appearing on the 6th Chinese Bioindustry Conference. Science and Technology of Food Industry, 2012,33(16):I0004-I0004.

[5] Zhang Shujun, Zhang Yanli. Analysis on European Marine Biotechnology Development Strategies and Inspirations on China. Advances in Marine Science, 2012,30(3):450-456. 